\title{
A scientist walks into a bar
}

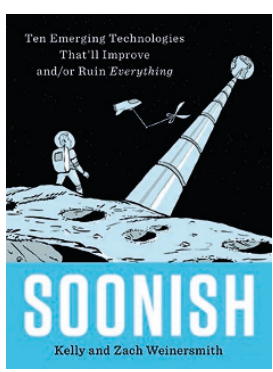

Soonish

by Kelly and Zach

Weinersmith

PARTICULAR BOOKS:

2017. 368 PP. $€ 18.99$

magine, after a long week at work, you are sitting in a bar. You're reminded of your undergraduate days, when you could spend hours on a night out with your friends discussing the plausibility of your favourite science fiction shows, so you decide to strike up a conversation with the person next to you and ask them what they think the future might hold. If you're lucky, you are sitting next to Kelly Weinersmith, a parasitologist in the BioSciences Department of Rice University, or her husband Zach, the artist behind the webcomic Saturday Morning Breakfast Cereal (SMBC). Not only would they be happy to engage in a conversation about the world of tomorrow, they'd also have done the necessary background research to make their predictions more than mere speculation. In their new book, Soonish, they predict the technologies that are likely to become part of our lives in the near future using precisely this barfly scenario as a starting point.

The Weinersmiths dug through the literature and interviewed a number of researchers working on aspects of the ten different topics they discuss to find out how close we really are to space travel and designer babies - and why we don't have fusion-powered toasters yet. In answering these questions (and to make their predictions realistic) they go beyond examining the state of research alone and also take into account economic factors, as well as the ethical considerations that could or should still prevent viable technology from becoming reality.

Soonish begins with a nice example: humankind's enduring fascination with journeying to the stars. The sheer cost of leaving Earth - currently around US $\$ 10,000$ per kilogram - is the biggest barrier to space exploration and even more so to space tourism. And so the book explores the question of how access to space could become affordable in the near future, and how asteroid mining might make these efforts useful from a practical perspective.

It then moves on to the objects that might be part of everyday life in the future. In fact, the Weinersmiths suggest that we might have to completely rethink our idea of an object. Shape-changing smart materials, if taken to an extreme, might leave us all in possession of a 'bucket of stuff', a term that Dr David Duff from the Palo Alto Research Center has coined for an undefined sludgelike substance that can take the shape of any item.

Finally, they ask how technology will change our bodies when personalized medicine or bioprinted organs become available, or when our interaction with machines is extended by computer-brain interfaces. The Weinersmiths divide these into three increasingly unpleasant categories: non-invasive interfaces, 'pretty darn invasive' interfaces and 'really superinvasive' interfaces - essentially wires stuck into the brain.

This classification is a typical example of how the authors stay true to their premise of discussing science from a barstool. They even slip in a collection of anecdotes reflecting the authors' experiences in researching and writing the book. These come in

\section{HOW NERDS AND PROTONS WORK}
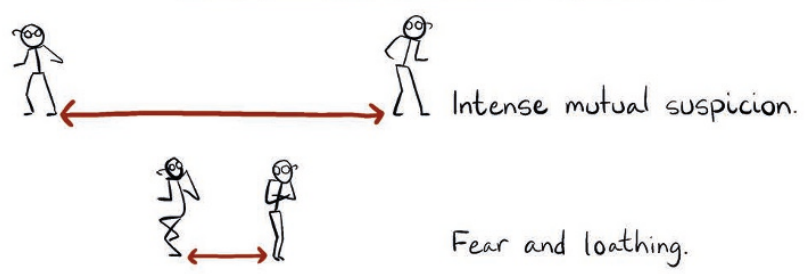

the form of numerous (but humorous) footnotes, a number of comic illustrations, and a nota bene at the end of each chapter, containing an additional story or fact that the Weinersmiths discovered when researching the topic at hand. They are adept at analogies to make abstract theories more comprehensible for a general audience. My personal favourite was the description of the distance-dependent forces involved in nuclear fusion by way of matchmaking between nerds (pictured), which bears a certain similarity with the famous cocktail-party analogy for the Higgs boson - a similarity I choose to believe was intended by the authors.

Soonish is a thoroughly enjoyable read, especially for those who don't mind that the Weinersmiths' (generally wellfounded) speculation sometimes goes off on a tangent. However, I have to admit that their casual style began to grate on me towards the end of the book, as did the sometimes repetitive references to popular culture, such as the robot uprising in 2027. Nonetheless, the science itself, and the potential of technologies such as human-machine interfaces, were more than enough to keep me turning the pages. This was probably helped by the progression of topics from physics-heavy subjects like space elevators and fusion to less familiar fields like biotechnology, which tend to be more fascinating to the average physicist.

Naturally, a book about the future does not stop at presenting the current state of the art and so the reader is invited to join the Weinersmiths in imagining how such technologies might change their lives - for better or worse. As well as dreaming up the gadgets they might one day be given for Christmas, they also make a serious effort to discuss the potential risks of even the most beneficial developments. However, their excitement about the technologies to come and equally about the science that could enable them far outweighs these concerns, so the reader will close the book with anticipation rather than a serious fear of the robot uprising. Soonish may not offer a dewyeyed vision of the near future, but at a time when dystopian fiction is the latest trend, it certainly makes for a pleasant exception. 